\title{
COMPARISON OF SELF-REPORTED AND COMPUTER-BASED HAZARD PERCEPTION SKILLS AMONG NOVICE AND EXPERIENCED DRIVERS
}

\author{
Nebi Sümer \\ Department of Psychology \\ Middle East Technical University \\ Ankara, Turkey \\ E-mail: nsumer@metu.edu.tr \\ Ayça Berfu Ünal, Ahmet Birdal, Pınar Çınar, and Sema Çevikoğlu \\ Psychotechnical Systems Group \\ Meteksan Sistem A.Ş. \\ Ankara, Turkey
}

\begin{abstract}
Summary: Hazard perception (HP) ability of novice drivers has been studied mainly by using computer-based implicit measures, such as simulators or video clips. In this study, we tried to replicate and extend Farrand and McKenna's (2001) study that compared computer-based HP testing with self-assessment. We measured HP latencies of experienced and novice drivers by using video clips reflecting actual traffic scenes and their domain-specific self-assessment. Participants (43 novice and 65 experienced drivers) were administered the computer-based Turkish Hazard Perception Test and a brief self-reported HP scale. Results indicated that experienced drivers had significantly shorter reaction time than novice drivers on computer-based video clips with a small effect size, but they reported much better HP skill on paper and pencil test with a strong effect size. Although the computer-based test scores were not correlated with selfreported HP for the novice driver group, they were negatively and significantly correlated for the experienced driver group, suggesting that experienced drivers develop a stronger overconfidence effect in their driving and hazard perception skills than novices.
\end{abstract}

\section{INTRODUCTION}

Effective scanning of the traffic environment, detecting potential hazards quickly, and anticipating a wide range of other road users' behavior are critical processes in preventing potential crashes. Hazard perception (HP) is a higher order cognitive ability that regulates these processes for driving safety. Crick and McKenna (1992) simply defined HP "as the ability to identify potentially dangerous traffic situations." Past research demonstrated that, as compared to experienced drivers and/or driving experts, novice drivers fail in optimally scanning the road, detecting the potential hazards, and taking the most effective actions to prevent crashes due to their undeveloped driving skills, schemas, and limited anticipation experiences (Deery, 1999; Gregersen \& Bjurulf, 1996; Grayson \& Groeger, 2000). In a recent study, Wallis and Horswill (in press) showed that for novice drivers, a higher threshold of danger must be present before they detect a hazardous situation than for experienced drivers. Because of these limitations, young and novice drivers have substantially higher accident risk than experienced drivers. For example, young (18-19 year old) drivers are involved in accidents five times more than older (35-50 year old) drivers in Sweden (Engström, Gregersen, Hernetkoski, Keskinen \& Nyberg, 
2003). Considering the critical role that hazard perception ability plays in transport safety, it is imperative that HP ability of novice drivers should be objectively assessed and the measures for HP should be experimentally validated.

In previous studies, HP ability has been typically measured via implicit measures by presenting drivers with filmed footage of various traffic situations, including hazards or traffic conflicts, and photographs of traffic scenes, or scenes in driving simulators, and asking them to respond when they detect a potential hazard, usually by pressing a button (e.g., McKenna \& Crick, 1991). Calculated reaction time to each hazard is used as the indication of hazard perception latency. Studies comparing novice and experienced drivers usually showed that novices have higher reaction time scores than experienced drivers. For example, Sexton (2001) found that there were significant mean differences between the HP scores of learner, novice, and experienced drivers, with the experienced drivers scoring the best, followed by novice drivers, and learners.

Although experienced and novice drivers differ in HP latency measures on computer or simulator-based reaction time measures, it is not well known if their self-assessment of hazard perception ability corresponds to computer-based latency scores. Farrand and McKenna (2001) examined whether self-reported perceived driving ability was related to video-based hazard perception latencies. They did not find a significant relationship between self-assessed driving ability and hazard perception scores. However, it was found that female drivers rated the situations as more hazardous and rated themselves lower in ability in dealing with the hazards than male drivers. In short, subjective assessment of driving skills was shown to be unrelated to actual performance, since females were as good as male drivers in detecting the hazards. We aimed to replicate Farrand and McKenna's study by emphasizing three major points. First, instead of directly measuring perceived hazard perception ability, Farrand and McKenna measured perceived driving skills and accident risk. We directly asked about hazard perception skills on the road rather than measuring general driving skills. Second, Farrand and McKenna used scenario-based videotapes of road scenes including hazards; we used video clips of traffic flows, including road hazards and conflicts recorded in real-life traffic environment. Finally, Farrand and McKenna did not compare novice and experienced drivers (they compared male and female drivers) on the basis of computer-based and self-reported HP ability. We aimed to compare novice and experienced drivers on both measures.

\section{METHOD}

\section{Participants}

Initially, 185 drivers participated in the study; however, 46 drivers who could not be classified under the experienced or novice groups due to their driving history and 31 female drivers who were mainly classified in the novice group were excluded from further analyses to have clear categorization on the driving experience. Therefore, main analyses were conducted on the remaining 108 drivers (43 novice and 65 experienced). Drivers who were actively driving less than a year or who have been recently licensed were categorized under the novice driver group, and those who were actively driving more than 3 years and whose weekly km driven was over $200 \mathrm{~km}$ were categorized as experienced drivers. The mean age of the drivers was 28.42 (SD = 7.59). 


\section{Materials}

Turkish Hazard Perception Test (T-HPT). The T-HPT is a computer-based test of HP developed for the purpose of generating a reaction time latency measurement consisting of the common road hazards and conflicts in Turkey, based on actual traffic scenes. As in the previous HP tests, recording was done from the driver's point of view usng a camera stabilized on the windshield of a car. All recordings were taken from real traffic flows in major cities of Turkey. A total of 266 scenes were selected by creating clips that varied between $20-40$ seconds in length by examining the video-footage. Initially, the constructed clips were classified under broad categories by using the Annual Traffic Statistics about the causes of accidents in Turkey, such as hazards occurring in junctions, overtaking hazards, close-following hazards, etc. These items were further examined and rated by a panel using a 10-point Likert-type scale $(10=$ very hazardous, $1=$ not hazardous), and 27 items with the highest ratings were identified as the test items. Initially a pilot study was conducted to test the discriminating power of the hazard clips and to receive feedback about the inter-phase of the test, the instructions, and trial sessions. Items that were found to be inefficient in discriminating between novice and experienced drivers were excluded.

The final version of the T-HPT consisted of 18 real-life traffic scenes. Sixteen clips included hazards and two clips did not contain any hazards and served as control items. Within the hazardous scenes, four clips included two hazards which were not overlapping in the occurring time. As can be seen in Table 1, six of the hazards were developing ones from the joining lane. These hazards consisted of violations of the right to pass, such as a car suddenly pulling in front of the car. Six of the hazards occurred in the opposite lane and these hazards consisted of faulty overtaking of the car in the opposite lane. The remaining hazards occurred in the driver's own lane and they contained pedestrians or bicyclist/motorcyclist jumping into the road, as well as other cars. The hazards that were caused by other vehicles in one's own lane mostly consisted of other drivers' sudden maneuvering or faulty lane changing.

It was clearly explained to drivers what a hazard means in traffic and how they could respond by pressing a button connected to a computer; three trial clips were used for demonstration purposes. If drivers failed to understand how to respond to a hazard, trials were repeated until they fully mastered the instructions. Drivers were allowed to give five responses at most. Hazard response windows were created tapping the onset and offset of the hazards. The scoring of each response (reaction time) was done with reference to where it was positioned in the hazard response areas. These scores were further converted into t-scores to standardize each item's score, and a mean hazard perception score was obtained using T-scores.

Table 1. Characteristics of Hazards

\begin{tabular}{|l|l|c|}
\hline Joining Path & \multicolumn{2}{|c|}{6} \\
\hline Opposite Lane & \multicolumn{2}{|c|}{6} \\
\hline \multirow{4}{*}{ Own Lane } & Cyclist/Motorcyclist & 1 \\
\cline { 2 - 3 } & Pedestrian & 4 \\
\cline { 2 - 3 } & Cars & 2 \\
\cline { 2 - 3 } & Animals & 1 \\
\hline \multirow{2}{*}{ Total \# of hazard } & \multicolumn{2}{|c|}{20} \\
\hline
\end{tabular}


Self-Reported Hazard Perception Scale (SRHP). A short scale consisting of six items was developed for self-reported assessment of hazard perception skills. Items were: 1 . There are times that I find it hard where to attend in traffic; 2. I have difficulties in detecting hazards on the roads that I did not drive before; 3 . I can easily recognize the vehicles that create hazards and risk in traffic; 4. I can foresee what kinds of dangers I could face just by looking at the traffic flow; 5 . I can predict what kinds of dangers there are at what types of roads; 6 . It is difficult to predict for me beforehand how other roads users can act. Participants were asked to indicate how often they experience the situations that were given in the items using a 6-point Likert-type scale $(1=$ never, 6 = always). Items were recoded appropriately so that higher scores correspond to better hazard perception ability. Due to the principle component analysis, a single factor structure emerged explaining $51 \%$ of the variance. Item-total correlations ranged between .45 and .67 and Cronbach's alpha for the scale was satisfactory $(\alpha=.80)$.

\section{Procedure}

Participants were first registered on the computer by entering the personal and driving-related information. Following registration, they completed the self-reported hazard perception scale on the computer. Then, they were given a 10-minute long multimedia presentation on the screen related to the hazard perception test. The responses to self-reported and computer-based hazard perception tests were recorded in the database and then converted into a text-file to be used in analysis.

\section{RESULTS}

First, two separate ANCOVA analyses were run controlling for the effects of age - one for the computer-based hazard perception (CBHP) scores and the other for self-reported hazard perception (SRHP) scores. The results of the ANCOVA on the CBHP scores revealed that on average, experienced drivers had significantly shorter reaction times than novice drivers $(F$ $(1,105)=6.20, p<.01)$. However, although experienced drivers scored significantly higher $(M=$ $51.94, S D=.63)$ than novice drivers $(M=48.92, \mathrm{SD}=.84)$, as shown in Figure 1 , mean difference between driver groups was minimal, explaining only $6 \%$ of the accounted variance $\left(\mathrm{Eta}^{2} .=.06\right)$. The results of the ANCOVA on the SRHP also revealed that experienced drivers reported significantly higher $(M=4.73, S D=.11)$ hazard perception skills than novice drivers $(M=3.85, S D=.15)(F(1,100)=17.38, p<.001)$. The mean difference between novice and experienced groups was high as compared to CBHP. As can be seen in Figure 2, the distribution of the groups diverged with a relatively high discrimination $\left(\operatorname{Eta}^{2}=.15\right)$.

Second, correlations between the total scores of the CBHP and SRHP were calculated. There was no significant correlation between the two scores. However, when the within-group correlations were calculated separately for novice and experienced driver groups, the CBHP was negatively correlated with the SRHP for experienced drivers $(r=-26, \mathrm{p}<05)$. When the correlations between each CBHP item and the mean score of the SRHP scale were examined, it was found that the majority of the correlations were in negative directions, four of which were significant or marginally significant for experienced drivers. 
In terms of novice drivers, the findings showed that there was not a significant relationship between the CBHP scores and the SRHP scores ( $\mathrm{r}=.05$, ns.). When the correlations were examined separately for each item, it was seen that the pattern of relationships was mostly positive, with two correlations reaching to significance. Overall, it was concluded that the relationship between self-reported HP and hazard clips were weak for both novice and experienced drivers.

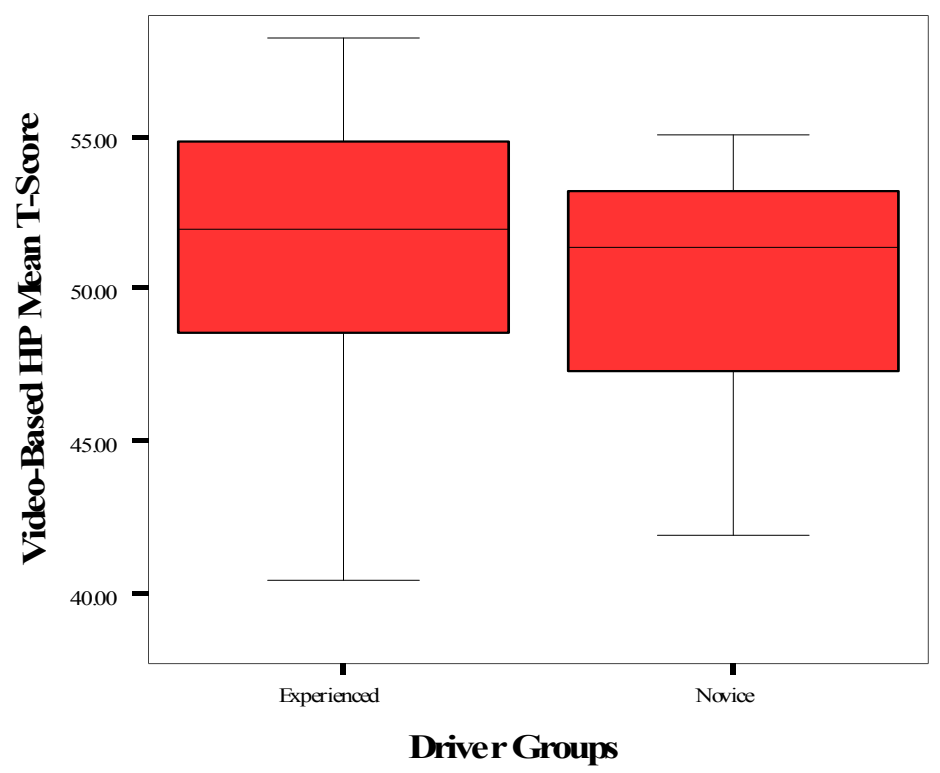

Figure 1. Mean response latencies on Computer-Based Hazard Perception

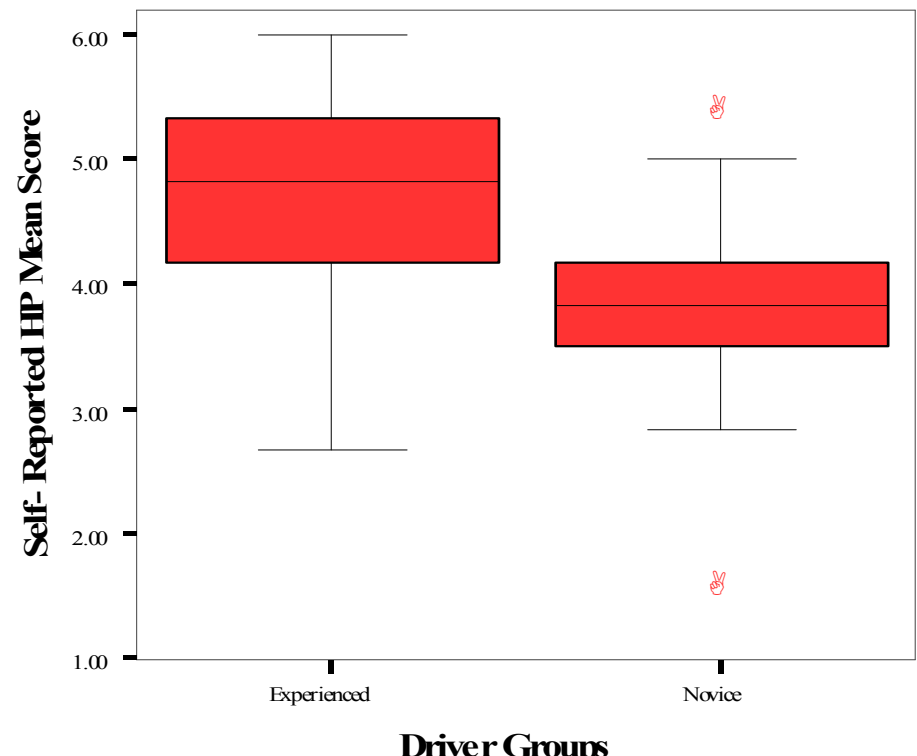

Figure 2. Mean scores on Self-Reported Hazard Perception 


\section{DISCUSSION}

This study aimed to question the consistency between subjective (self-assessed) and objective (computer-based) measures of hazard perception among a sample of novice and experienced drivers. Previously Farrand and McKenna (2001) showed that subjective assessments related to driving skills were not related to actual performance in hazard perception tests. Unlike their design, in the current study a short hazard perception scale was used rather than asking the participants to rate their competencies in dealing with the specific hazard shown in a clip. We found both novice and experienced drivers had higher scores in both types of measurements with a clear discrimination in self-report as compared to computer-based measure. However, consistent with Farrand and McKenna's finding, overall these two types of measurements were not significantly correlated in the current sample. Unexpectedly, we found that there was a significant negative correlation between CBHP and SRHP scores for experienced drivers, suggesting an overestimation in hazard perception skills in the self-reported measure. This may reflect an overconfidence effect about their driving and hazard perception skills for experienced drivers, which should be examined more in further studies.

In previous studies, young and novice drivers reported high overconfidence in their driving skills (Finn and Bragg, 1986; DeJoy, 1992). However, it seemed that this tendency was not prevalent when asked about specific hazard perception skills. The items of the self-report measure in the current study tapped the higher-order skills, which are closely connected to hazard anticipation, road reading, situational awareness or scanning strategies. Therefore, it is likely that novice drivers were able to make more realistic assessments when asked about domain-specific skills. Besides this, the novice driver group in this study mainly consisted of drivers who had been recently licensed with a very low level of driving practice. Supporting Groeger and Grande's (1996) assumption suggesting that overconfidence increases with driving experience, in the current study, experienced drivers reported more confidence in their skills than novices. A follow-up study investigating short-term change in HP skills as drivers gain experiences in driving may reveal the critical time at which the overconfidence effect emerges.

\section{ACKNOWLEDGMENTS}

This study was supported by The Scientific and Technological Research Council of Turkey, Research \& Development Support Program (TUBITTAK TIDEB) and Meteksan Sistem A.Ş. We would like to thank Ömer Çetin Sudan for his contribution in selecting the video clips and accessing the novice drivers.

\section{REFERENCES}

Crick, J., \& McKenna, F.P. (1992). Hazard perception: Can it be trained? Proceedings of Manchester University Seminar: Behavioural Research in Road Safety II.

Deery, H.A. (1999). Hazard and risk perception among young novice drivers. Journal of Safety Research, 30, 225-236.

DeJoy, D. M. (1992). An examination of gender differences in traffic accident risk perception. Accident Analysis and Prevention, 24, 237-246. 
Engström, I., Gregersen, N. P., Hernetkoski, K., Keskinen, E., \& Nyberg, A. (2003). Young novice drivers, driver education and training. VTI rapport 491A. Retrieved from the Web August 25, 2005. http://trb.org/news/ blurb_detail.asp?id=1926.

Farrand, P, \& McKenna, F.P. (2001). Risk perception in novice drivers: the relationship between questionnaire measures and response latency. Transportation Research Part F 4, 201-212.

Finn, P., \& Bragg, B.W.E. (1986). Perception of the risk of an accident by young and older drivers. Accident Analysis and Prevention, 18, 289-298.

Grayson, G.B., Groeger, J.A. (2000). Risk, hazard perception and perceived control. In: Proceedings of the DTRL Novice Drivers Conference, 1-2 June, 2000.

Gregersen, N.P. \& Bjurulf, P. (1996). Young novice drivers: towards a model of their accident involvement. Accident Analysis and Prevention, 28, 229 - 241.

Groeger, J.A., \& Grande, G.E. (1996). Self-preserving assessments of skill? British Journal of Psychology, 87, 61-79.

McKenna, F.P., \& Crick, J.L. (1991). Hazard perception in drivers: a methodology for testing and training. Final Report. Transport Research Laboratory, Crowthorne, UK.

Sexton, B. (2001). Development of hazard perception testing. Proceedings of the Novice Drivers Conference. 26 July 2001, UK. Retrieved from the Web July 10, 2005. www.dft.gov.uk/stellent/groups/dft_rdsafety/documents/page/dft_rdsafety_611030.hcsp.

Wallis, T.S.A., \& Horswill, M.S. (In press). Using fuzzy signal detection theory to determine why experienced and trained drivers respond faster than novices in a hazard perception test. Accident Analysis and Prevention. 